\title{
Face Tracking and Recognition using Matlab and Arduino
}

\author{
A. Guru Sai Sasidhar, P. Jagadeesh
}

\begin{abstract}
In this paper, we propose topologyprotectivediagram coordinative (VIOLA/JONES) strategy for halfway face acknowledgment. In any case, faces in true free is also stopped up by objects or absolutely fully completely different faces, that cannot provide the whole face footage to depiction, Key purpose primarily based fragmentary face acknowledgment ways in which to handle this, our VIOLA/JONES technique evaluates a non inflexible amendment secret writing the second organize geometric structure of the diagram, with the goal that more precise put together, powerful correspondence are visiting be dotted with the topological information so camera capture the face whether or nor matched or unmatched the image, if the matched the mortal you get the buzzer otherwise you can get semiconductor contraption lightweight watch the Arduino uno. We tend to propose a topology safe guarding helper coming up with (viola jones) strategy near develop the subsequent request structure for every face.
\end{abstract}

Keywords: matlab software, arduino

\section{INTRODUCTION}

Programmed appearance investigation which incorporates, e.g., face discovery, face acknowledgment and facial articulation acknowledgment has turned into a operational theme in PC vision inquire about . A crucial concern in face examination is finding gainful descriptors aimed at face look. One of a kind widely inclusive systems, for instance, Main Segment Investigation (PCA), Straight Discriminant Examination (LDA) and the later 2-D PCA have been considered generally yet of late too nearby descriptors have picked up consideration because of their strength to difficulties, for example, existing furthermore, enlightenment variations. This paper displays a novel descriminator dependent on nearby twofold design surface acmes separated from nearby facial locales. In Neighborhood Highlight Examination , bits of adjacent spatial assistance are used to isolate information about neighborhood facial parts. Adaptable Pack Diagram Managing (EBGM) delineates appearances using Gabor direct rejoinders in confident makeover places of interest and a grid depicting the latitudinal kindred of these tourist spots. In which a part based face acknowledgment framework plainly beat worldwide methodologies on a assessment data containing faces pivoted inside and out. Utilizing neighborhood photometric highlights for item acknowledgment in the more broad setting has turned into a generally accredited methodology. Discovering great discriminators aimed at the presence of neighborhood makeover districts is an open problem. In a perfect world, these descriptors to be anything but difficult to register then consume tall additional session change (i.e., between various people on version of face acknowledgment) what's more, intra class fluctuation, whose implies that the discriminator ought to stay vigorous by regard to maturing of the subjects, substituting enlightenment plus different aspects However, face acknowledgment issue has not been related to that advancement in surface examination field as it has not been examined from such perspective .In this paper, we give a more nitty gritty examination of the planned portrayal, existing extra outcomes then talk about extra .Hiding information definitely wrecks the host picture despite the fact that the bending acquainted by stowing away is intangible with the human visual framework. Be that as it may, there are some touchy pictures where any implanting bending made to the picture is insufferable, for example, military pictures, restorative pictures or fine art protection... Article location and subsequent are essential trendy plentiful PC vision applications counting movement acknowledgment, car wellbeing, and observation. A clear face following system is made by disconnecting the accompanying issue into three separate issues: Face identification in the casing, Initial facial highlights utilized for following, Face Tracking. Face identification in MATLAB should be possible utilizing a wide range of existing calculations. These calculations utilize diverse systems to distinguish the facial highlights. Some utilization edge

recognition strategies while some utilization differentiate partition. One of the generally utilized calculations is ViolaJones calculation

Revised Manuscript Received on August 14, 2019.

A. Guru Sai Sasidhar, Department of Electronics \& Communication Saveetha School Of Engineering(SIMATS), Chennai,Tamilnadu,India. (Email: sasidhar525@gmail.com)

P. Jagadeesh, Department of Electronics \& Communication

Saveetha School Of Engineering(SIMATS), Chennai,Tamilnadu,India

(Email: jagadeeshp@saveetha.com) 


\section{THE IMAGE PROCESSING SYSTEM}

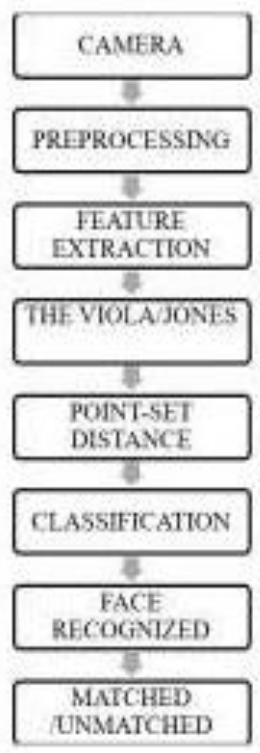

Hardware Section

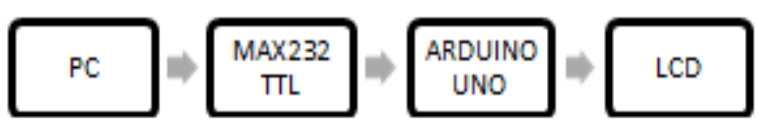

\section{FIG. 1 Square Outline OF Picture Handling Framework}

\section{Preprocessing:}

Informational indexes can require preprocessing strategies to guarantee precise, effective, or important investigation. Information cleaning alludes to techniques for discovering, evacuating, and supplanting terrible or missing information. Distinguishing nearby extrema and sudden changes can recognize huge information patterns. Smoothing and detrending are forms for expelling commotion and direct patterns from information, while scaling changes the limits of the information. Gathering and binning strategies are procedures that recognize connections between the information factors.

\section{Feature Extraction:}

In model avowal and in portrait dealing with, feature abstraction is a outstanding kind of dimensional decline. Right after the facts to a count is extremely tremendous to potentially be taken overhaul of and it is distrusted close be largely tedious, by at that point the data information will be changed into a reduced portrayal set of highlights. Changing the information into the approach of highlights is called fuse extraction. If the features removed stay warily select it is classic that the features fixed will expel the critical evidence from the data to play out the perfect duty using this contracted delineation instead of the complete size data. Feature mining incorporates streamlining the proportion of benefits mandatory to show an enormous plan of data correctly. When execution check of composite data one of the difficult questions devises from the amount of viewpoints included .Examination thru endless generally requires a great deal of memory and count control or a gathering guesstimate which over fits the arrangement trial and totals up deficiently to new models. Feature abstraction is a universal term aimed at procedures for structure mergers of the variables to get everywhere these questions whereas so far depicting the data per sufficient exactness.

\section{Viola/Jones Algorithm:}

Identification Face from Picture and spare trimmed face in an organizer. Basic picture: a quick method to register basic "highlights". In Adaboost the feeble student is only a component selector. The favorable position is that if there are $\mathrm{N}$ frail students there are just $\mathrm{N}$ highlights to process. Fell blend of classifiers. The greater part of genuine negatives are dismissed exceptionally quick at the at the initial couple of stages. Can keep high discovery rate and low false positive rate

\section{Classification}

It is an item which characterized through a standard choice of its neighbors, with the assurance alloted event for most common class among its. Traditionally Euclidean separation is utilized as the separation metric; in any case, this is appropriate for unlimited factors. In such circumstance as the arrangement of content, elective measurement, crossing point metric or Hamming separation can be utilized.

\section{MAX232TTL:}

The MAX232 is a twofold driver/recipient that integrat a capacitive energy originator to stream RS 232 energy stages since a singular $5 \mathrm{v}$ supply. Each inheritor adherents RS-232 to $5 \mathrm{v}$ TTL/CMOS stages. Each chauffeur vagaries concluded TLL/CMOS info stages hooked on EIA-232 measurements. The P3_0 (RX) and P3_1 (TX) stick of controller is related through the greatest 232 driver and the TX and RX rod of max 232 is related with the GSM to the modem and PC

\section{ARDUINO UNO Design}

Arduino/Genuino Uno is a microcontroller panel subject to the ATmega328P. It has 14 automated data/crop bits (of which 6 can be used as PWM bits), 6 straightforward information sources, a $16 \mathrm{MHz}$ quartz valuable grit, a USB affiliation, a clout knave, an ICSP title and a retune clasp. It contains all projected to help the microcontroller; mainly line it to a PC with a USB connection or power it with an airborne conditioner to-DC connector or series to start. You can interfere with your UNO deprived of focusing on much terminated achieving something mistakenly, maximum desperate result possible you can ancillary the chip aimed at several bucks then start by and by yet again.

\section{LCD}

LCD (Liquid Crystal Display) screen is an electronic presentation unit and locate a wide degree of employments. A 16x2 LCD display is central unit and is all everywhere

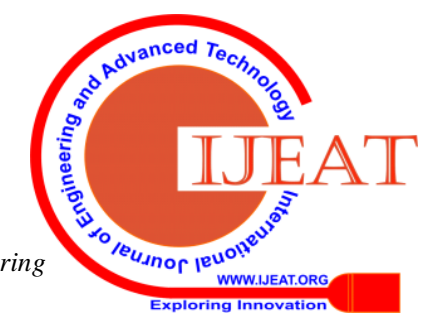


ordinarily utilized in different devices and circuits. These units are upheld more than seven zones and extra multi part LEDs. The reasons being: LCDs are capable; feasibly programmable; have no obstruction of showing up and even routine charms (not in the slightest degree like in seven sections) action, and so forth.

\section{OBJECTIVE}

The fundamental goal of the task is to recognize a human face in each edge originating from a web camera and the caught picture is prepared utilizing viola- jones calculation utilizing MATLAB and to identify the countenances and send signs to the Arduino board to control the development of the camera utilizing two servo engines.

\section{EXISTING SYSTEM}

Existing habits through which for acknowledging face affirmation between the closeness of harvest are supported the intricacy show and cannot manage non-uniform yield variations that a great part of the time rise up out of inclines and turns close by said Picture. Lighting up due precipitous deviations has been stacked as a sophistication with one edification bit, and along these lines the illumination is believed to uniform over image. Topology cause set similar(TPSM) we've a convex to intend a topology verifying assistant organizing (TPSM) theory to construct a additional stuck solicitation edifice for each face and measure the change. Exploratory results proceeding four varied used look datasets demonstrate that our strategy defeats furthermost prevailing unique face affirmation systems. this, our TPGM strategy evaluates a nonunbendable change coding the second solicitation geometric configuration of the diagram, thusly a great deal of right and amazing communication will figured with the topology data an image part dreadfully very light image are often a weighted standard of the pixel's neighborhood within the key sharp image. In like manner, turn out is sculptural as a convolution task between the fundamental image and a turn out channel bit that addresses the hundreds....

\section{EXISITING SYSTEM DISADVANTAGES}

1. Memory intensive.

2. Sensitive to initialization, outliers.

3. It is not suitable for very noisy and edgeless images.

4. It is not suitable for images whose boundaries are very smooth.

\section{PROPOSED SYSTEM}

Another halfway face acknowledgment way to deal with recognize people of eagerness from their fractional countenances. Given a unification of display picture and test face fix, we will in general beginning notification key concentrations and essence their nearby textural choices. outline to discriminatively organize these 2 isolated neighborhood abilities, any place each the textural and geometrical learning of nearby judgments are unequivocally used for planning at the unclear time. The VIOLA/JONES equation a individual's will attempt this just, anyway a Terminal precise captions and limitations. to mark the errand heaps of functional, Viola Jones wants full inspect fore straight countenances. in this manner on be identified, the entire face must aim to the camera and cannot be angled to any side. whereas it shows up these limitations may decrease the calculation's utility reasonably, as an outcomes of the distinguishing proof development is most much of the time pursued by an affirmation adventure, in seek after these purposes of restriction on cause are acceptable. technique to discriminatively organize these 2 expelled nearby capacities, any place individually the textural and geometrical facts of neighborhood judgments remain expressly used aimed at planning at the indistinct time. Strategy of article location works best for items that show non-repeating surface models, that effort to caliber bouts. this procedure be situated on its pith to figure well for reliably shaded things, for articles containing emphasis plans. Annotation that this condition is expected for investigating a precise article. Hip accounts of moving stuffs, one needn't make a difference object identification to each casing. Or maybe, one will use seek after plans alike the KLT condition to see conspicuous determinations amid the recognizable proof skipping boxes and trail their improvement amongst housings . we resolve when all is said in done will as a rule incline to offered nearly significant topics scheduled face affirmation.

\section{ADVANTAGES}

The sign of the RPSM is its strong coordinating plan, which considers both the Geometric circulation consistency and the textural similitude Constraint on the relative change is connected to keep from improbable face twisting.

\section{APPLICATIONS}

Facial affirmation. Face recognizable proof is used in biometrics, much of the time as a bit of (or together with) a facial recognition system. It is moreover exploited in video surveillance, human PC boundary and portrait database the boarded.

\section{RESULT AND OUTPUT}

This paper exhibits various picture preparing procedures to extricate face some portion of halfway face gives better upgrade of RGB to GRAY picture, Get include, Matched channel bolsters custering for Find Neighbor extraction through of face coordinated. With this technique, the utilization of coordinated channel can be investigation and thus guarantee a face acknowledgment. 

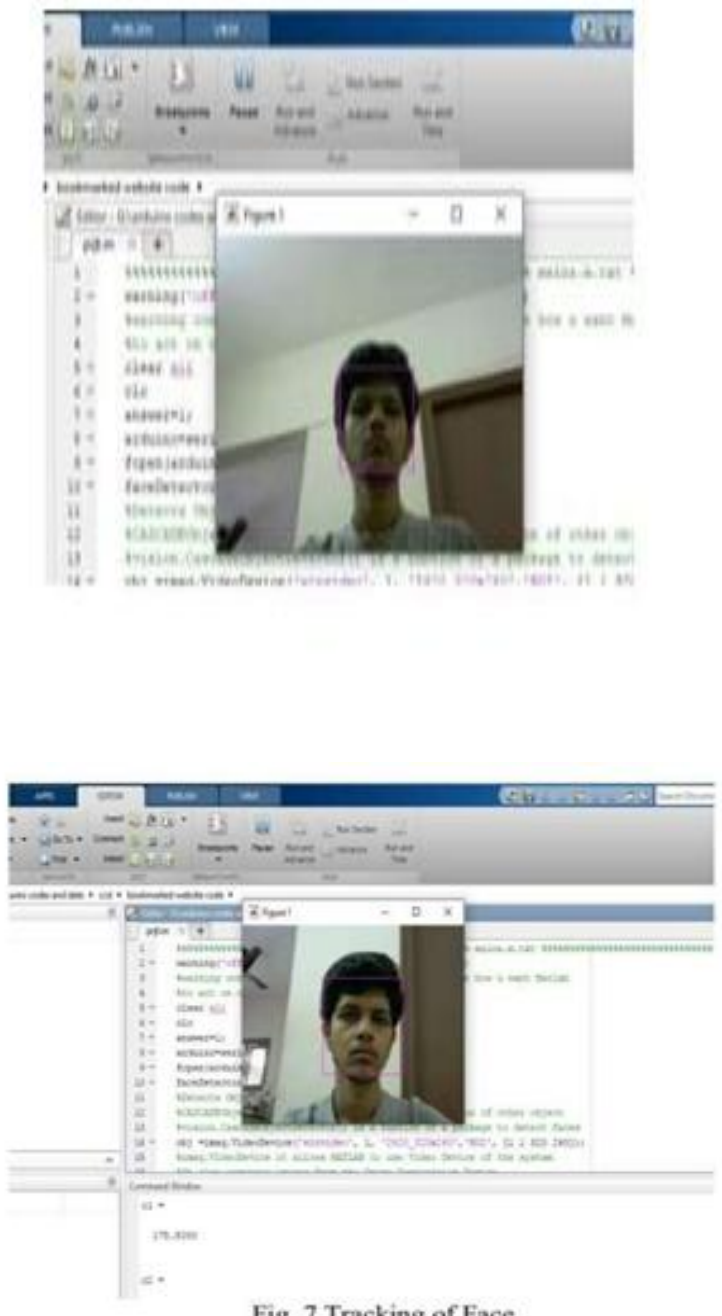

Fig. 7 Tracking of Face

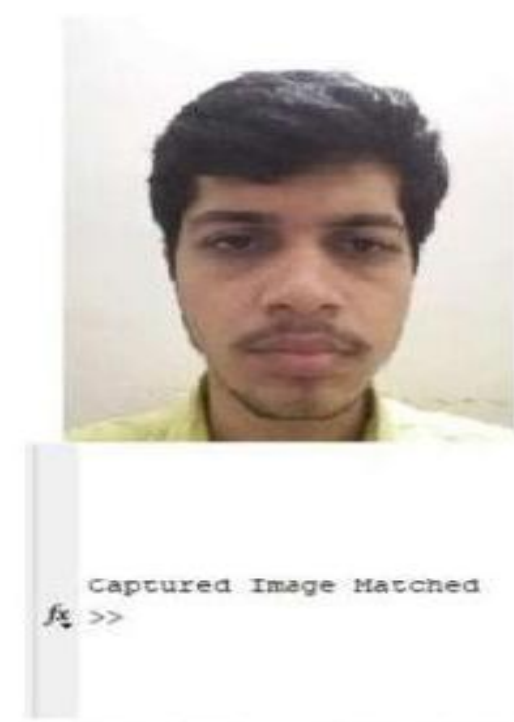

Fig. 8 Recognition of Face

\section{CONCLUSION}

In our work, the face position and following is mostly founded on the facial features. The count used for face area and following are Viola Jones figuring. It is a gainful and snappy computation when appeared differently in relation to various estimations. It is done by using MATLAB code, where Camera perceives the face and finishing is done the advancement of camera using a Microcontroller called ARDUINO. Resulting stage is face affirmation, where the recognized and pursued face is seen and it is facilitated it with available database-where set of pictures are starting at now open with different verbalizations and conditions. In case the match appears between apparent picture and picture open in database, by then it will show catch picture is facilitated else it gives catch picture isn't composed. We can execute this procedure in various spots where security and video perception is essential. In this paper we are kept with basic research focus conditions where the application part should be conceivable with stunning costs

\section{REFERENCES}

1. P.Viola and M.Jones,"Rapid Object Detection using a Boosted Cascade of Simple Features",conf. on comp. vision and pattern recognition 2001.

2. Jatin Chatrath, Pankaj Gupta, Puneet Ahuja, Aryan Goel and Shaifali M.Arora,"Real Time Human Face Detection and Tracking",International Conf. on Signal Processing and Integrated Networks

3. J. Connell, A.W. Senior, A. Hampapur, Y-L Tian, L. Brown, and S. Pankanti," Detection and Tracking in the IBM PeopleVision System",IEEE ICME, June 2004.

4. Edgar Osuna, Robert Freund, and F. Girosi," Training support vector machines: an application to face detection.",In Proceedings of the IEEE Conf. on Comp. Vision and Pattern Recognition, 1997.

5. C. Wren, A. Azarbayejani, T. Darrell, and A Pentland,"Finder: Real-time tracking of the human body", IEEE Trans. Pattern Analysis and Machine Intelligence, Vol.19, 1997, pp. 780-785.

6. D.Mikhaylov, A.Samoylov, P.Minin and A.Egorov,"Face Detection and Tracking from Image and Statistics Gathering",IEEE Conf. on Signal-Image Tech. and Internet-Based Systems.

7. Yang, Jian, et al.," Two-dimensional PCA: a new approach to appearance-based face representation and recognition", IEEE transactions on pattern analysis and machine intelligence, Vol.26.1, 2004

8. Ahonen, Timo, Abdenour Hadid, and Matti Pietikainen," Face description with local binary patterns: Application to face recognition", IEEE transactions on pattern analysis and machine intelligence, Vol.28.12, 2006

9. Belhumeur, Peter N., Joo P. Hespanha, and David J. Kriegman," Eigenfaces vs. fisherfaces: Recognition using class specific linear projection",IEEE Transactions on pattern analysis and machine intelligence, Vol.19.7, 1997

10. Gottumukkal, Rajkiran, and Vijayan K. Asari," An improved face recognition technique based on modular PCA approach", Pattern Recognition Letters, Vol.25.4, 2004 